\title{
Volcanisme Permien Du Massif De Chougrane-El Had Des Bouhsoussène (Maroc Central, Maroc)
}

\author{
Abdelaziz Boutsougame \\ Hassane Ouazzani
}

Faculté des Sciences Meknès, Département de Géologie, Zitoune, Meknès, Maroc

Hassan El Hadi

Université Hassan II de Casablanca, Faculté des Sciences, Laboratoire de géologie appliquée, Géomatique \& Environnement, Ben M'sik Sidi Othmane, Casablanca, Maroc

\section{Aâtika Eddif}

Centre régional des métiers de l'éducation

et de formation de Meknès, Maroc

doi: 10.19044/esj.2016.v12n27p81 URL:http://dx.doi.org/10.19044/esj.2016.v12n27p81

\begin{abstract}
Permian volcanic Chougrane-El Had the Bouhsoussène complex (central Morocco), manifested primarily by volcanic rocks rhyodacites nature and basalt intercalated in the clay-greso-conglomeratic permian deposits. These are rocks texture in general microlitic to porphyry. Basalts consists of a primary paragenesis formed of plagioclase, pyroxene, olivine and quartz and a secondary mineral assemblage consisting of muscovite, calcite, chlorite and quartz. The rhyodacites show primary paragenesis formed of plagioclase, pyroxene and quartz and a secondary mineral assemblage consisting of calcite and sericite. Petrographic characteristics of these rocks are comparable to those of the Permian volcanic complex Khénifra and Tiddas- Sebt Ait Ikkou (Central Morocco). Area Chougrane-El Had the Bouhsoussène created on intracontinentale tear in the Asturian phase (Michard, 1976) recorded three volcanic episodes, two major eruptive episode and a third in vein deposit mode.
\end{abstract}

Keywords:Permian volcanic activity, Chougrane El Had of Bouhsoussène, Central Morocco

\section{Résumé}

Le complexe volcanique permien de Chougrane-El Had des Bouhsoussène (Maroc Central), se manifeste essentiellement par des roches 
volcaniques de nature rhyodacites et basaltes, intercalées dans les dépôts argilo-gréso-conglomératiques permiens. Ce sont des roches à texture en général microlitique à porphyrique. Les basaltes sont constitués par une paragenèse primaire faite de plagioclase, pyroxène, olivine et quartz et d'une association minérale secondaire faite de muscovite, calcite, chlorite et quartz. Les rhyodacites montrent une paragenèse primaire composéee de plagioclase, pyroxène et quartz et une association minérale secondaire constituée de calcite et séricite. Les caractéristiques pétrographiques de ces roches sont comparables à celles des complexes volcaniques permiens de Khénifra et de Tiddas- Sebt Ait Ikkou (Maroc Central). La région de Chougrane-El Had des Bouhsoussène créée sur une déchirure intracontinentale lors de la phase asturienne (Michard, 1976) a enregistrée trois épisodes volcaniques, deux principaux et un troisième épisode éruptif à mode de gisement filonien.

Mots-clés : Volcanisme Permien, Chougrane- El Had des Bouhsoussène, Maroc Central

\section{Introduction}

$\mathrm{Au}$ Maroc, les séries permiennes sont conservées dans des bassins intramontagneux isolés et distants les uns des autres. C'est dans le Maroc Central que se produit l'essentiel des éruptions permiennes. La mise en place de corps magmatiques, de natures très variées, est contrôlée par la tectonique hercynienne (Cailleux et al., 1986). Les principaux volcanismes permiens du Maroc Central sont : volcanisme Bou Achouch, volcanisme de Chougrane-El Had des Bouhsoussène, volcanisme de Khénifra et volcanisme de TiddasSebt Ait Ikkou.

Le complexe volcanique permien de Chougrane-El Had des Bouhsoussène (Maroc Central) étudié ici n'a que rarement fait l'objet de levés géologiques détaillés. Les faciès de ce complexe volcanique sont des rhyodacites et des basaltes, intercalés dans les dépôts argilo-grèsoconglomératiques permiens. Ce sont des roches à texture en général microlitique porphyrique. La paragenèse primaire est principalement formée d'olivines, plagioclases, pyroxènes et quartz. La paragenèse secondaire comprend calcite, chlorites, opaques et kaolinite. Le magmatisme est largement représenté sous forme de coulées et filons.

Le but de ce travail consiste en l'étude pétrographique du complexe volcanique permien de Chougrane-El Had des Bouhsoussène et de le comparer aux volcanismes de même âge dans la région.

Pour faire cette comparaison, nous avons réalisé un échantillonnage de tous les faciès rencontrés sur le terrain pour une étude pétrographique complète. Une étude des épisodes volcaniques permiens du massif de 
Chougrane-El Had des Bouhsoussène sera également abordée.

\section{Contexte géographique et géologique du complexe volcanique étudié}

\section{Localisation du complexe volcanique}

Le Maroc Central ou massif hercynien Central (Termier, 1936) est défini comme un vaste quadrilatère dont Rabat, Azrou, Casablanca et Kasbat Tadla marquent les sommets.

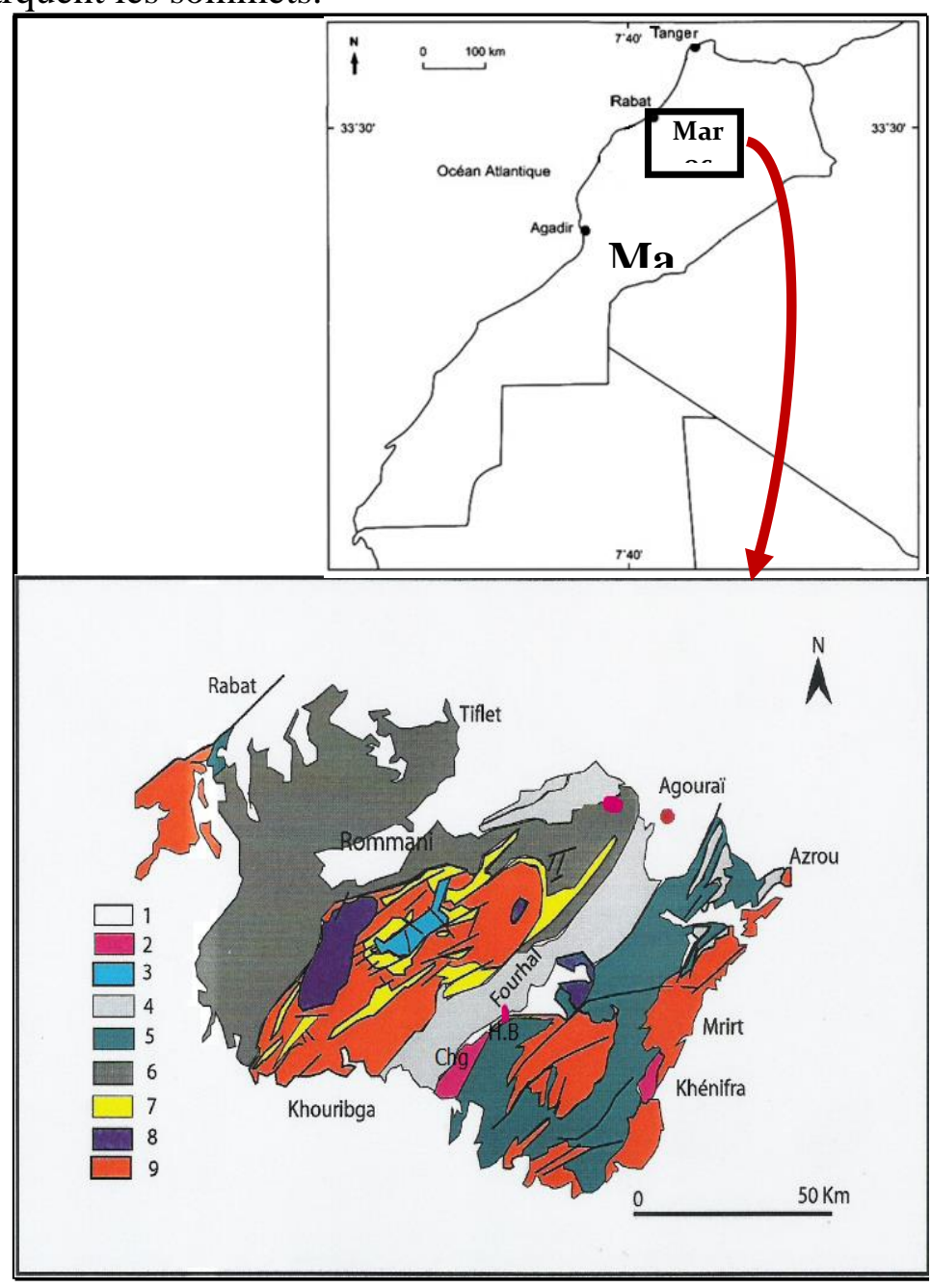

Figure 1 : Carte géologique du Massif Central (Bouya, 2014) avec localisation du complexe volcanique permien du bassin de Chougrane (Chg) - El Had des Bouhsoussène (H.B).

1) Formation post-paléozoïque, 2) Permien, 3) Namurien-westphalien, 4) Namurien inférieur, 5) Viséen, 6) Dévono-Dinantien, 7) Silurien, 8) granite tardi-hercynien et 9) paléozoïque inférieur (Cambrien à Silurien).

Le complexe volcanique du bassin permien de Chougrane-El Had des Bouhsoussène appartient à la partie méridionale du Maroc Central (fig. 1). 
Ce bassin est formé de deux parties qui sont géographiquement isolées : bassin de Chougrane $(20 \times 7,5 \mathrm{Km})$ et les affleurements restreints d'El Had des Bouhsoussène $(5$ x 1,5 Km).

\section{Contexte géologique}

Le complexe volcanique de Chougrane-El Had des Bouhsoussène est formé par des successions lithologiques différentes qui sont géographiquement isolées. La première; correspondant au bassin de Chougrane, montre deux générations de conglomérats et trois venues éruptives (les laves sont essentiellement des basaltes). Les conglomérats inférieurs, épais d'une dizaine de mètres, ne contiennent que des galets, de grès et de quartzites du socle. Alors que les conglomérats supérieurs renferment, outre des clastes du matériel éruptif sous-jacent, des éléments viséens. Au sommet, on note l'abondance de niveaux fins (marnes rouges et grès fins à ciment argilo-calcareux ou carbonaté). L'attribution de l'âge permien aux formations de Chougrane est fondée sur la datation géochronologique obtenue sur les formations volcaniques de Bir El Gassaa: $270 \pm 17$ Ma (Van Houten, 1976).

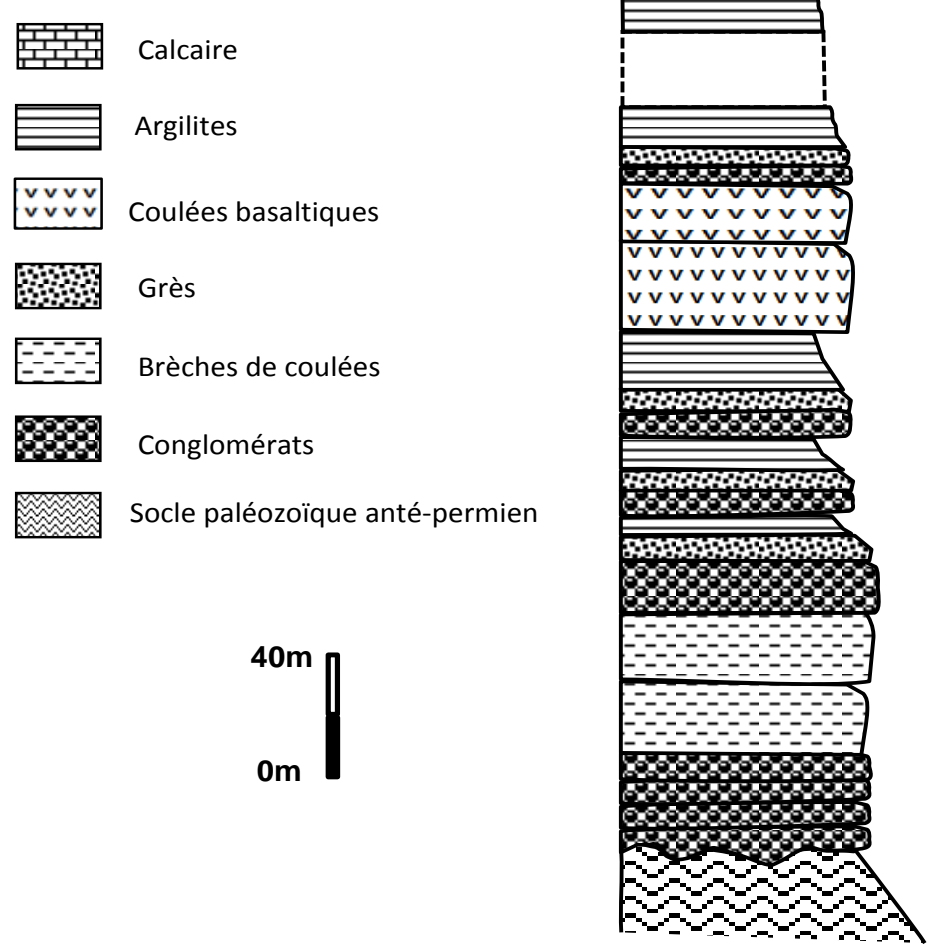

Figure 2 : Colonne lithostratigraphique des formations de Chougrane-El Had des Bouhsoussène 
Étude lithostratigraphique et pétrographique du bassin permien de Chougrane-El Had des Bouhsousène Étude lithostratigraphique

Deux coupes ont été levées dans deux zones séparées géographiquement ( $\mathrm{A}$ et $\mathrm{B})$

Figure 3 :

Schéma

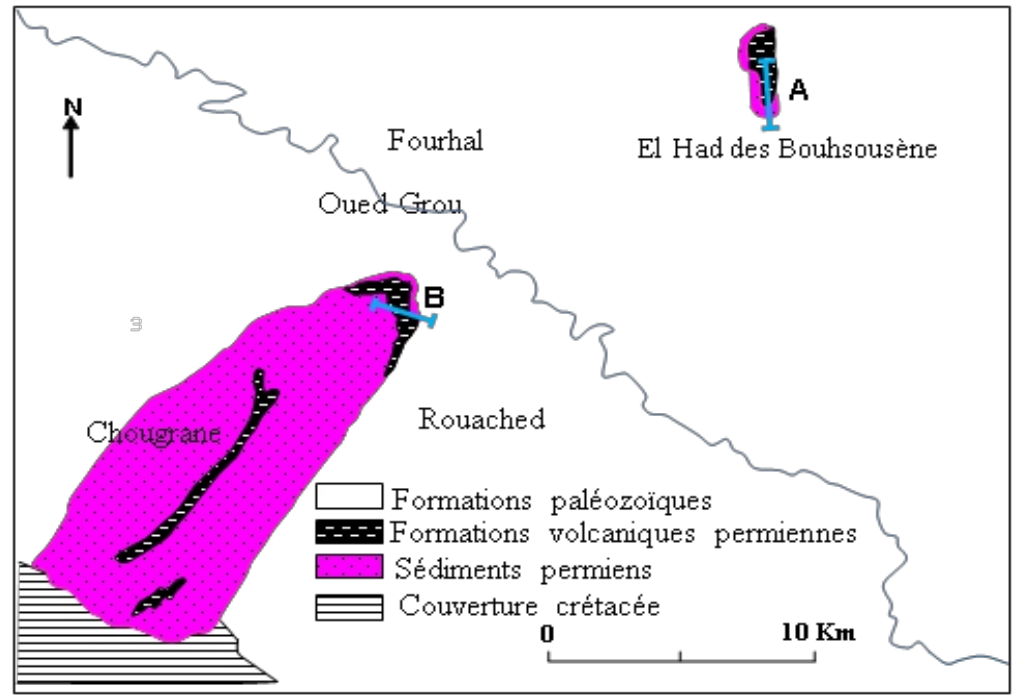

géologique du bassin permien de Chougrane-El Had des Bouhsousène (Cailleux et al. 1982, modifiée). A et B : emplacement des coupes levées.

\section{$\mathbf{S}$}

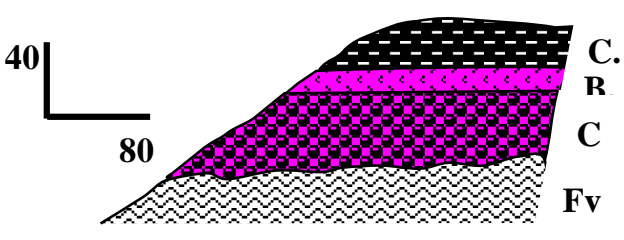

CoupeA, levée à Tazdot (Ouest du village de El Had des Bouhsousène)
C.v: Coulées volcaniques

B.v : Brèches volcaniques

Cg: Conglomérats Gr: Grès

Cg.s : Conglomérats supérieurs

Cg.i : Conglomérats inférieurs

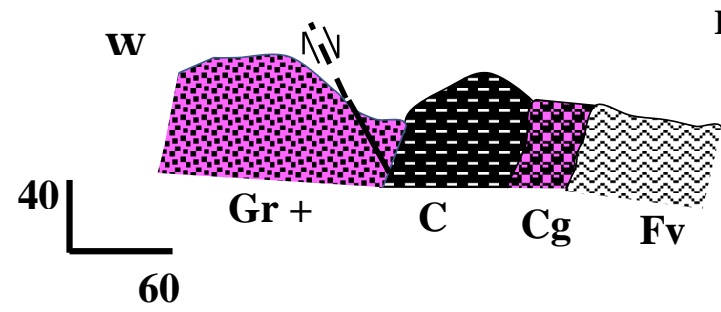

Fvn : FlyschoïdeViséo-

$\underline{\text { Coupe B }}$, levée au Nord Est du bassin de 


\section{Coupe A :}

Les conglomérats de base d'une puissance de $70 \mathrm{~m}$ reposent en discordance angulaire sur les flyschs viséens. Ces conglomérats sont composés de matériel terrigène grossier. Les éléments figurés, d'une taille maximum de $25 \mathrm{~cm}$, mal classés, anguleux ou émoussés selon la nature de l'élément, correspondent à des blocs et des galets de quartzites et grès ; ils sont enrobés dans une matrice argilo-sableuse. Ces conglomérats sont surmontés par des brèches volcaniques qui passent vers le haut à des coulées volcaniques puissantes de $40 \mathrm{~m}$ environ. L'étude pétrographique montre que les faciès volcaniques sont des rhyodacites.

\section{Coupe B :}

La formation inférieure est matérialisée par des conglomérats de base qui reposent en discordance angulaire sur les flyschs viséo-namuriens. Les conglomérats puissants de 10 à $15 \mathrm{~m}$ sont organisés en bancs indurés et composés de matériel terrigène grossier. Les éléments figurés, mal classés, peu imbriqués, anguleux ou émoussés selon la nature de l'élément, correspondent à des blocs et des galets de quartzites, de grès et pélites du socle viséo-namurien. Ils sont enrobés dans une matrice argile-sableuse. Les conglomérats de cette formation sont surmontés par une succession de coulées de basaltes puissantes de 50 à $60 \mathrm{~m}$. Une série détritique composée essentiellement de grès, d'argilites rouges et de conglomérats repose en discordance de ravinement sur les roches volcaniques basaltiques.

Le bassin permien de Chougrane est créé sur une déchirure intracontinentale lors de la phase asturienne, sur la bordure sud du Maroc Central (Michard, 1976 ; Cailleux et al., 1982).

On distingue trois épisodes volcaniques, deux principaux et un troisième épisode éruptif à mode de gisement filonien; ces épisodes sont séparés par des formations sédimentaires :

Le premier épisode volcanique est exprimé par des coulées rhyodacitiques qui surmontent en ravinement la formation sédimentaire inférieure. La formation inférieure puissante de $15 \mathrm{~m}$ environ est formée par des conglomérats de base qui reposent en discordance angulaire sur les flyschs viséens. Ces conglomérats, organisés en bancs, sont composés de matériel terrigène grossier et des éléments de socle. Les éléments figurés sont mal classés anguleux ou émoussés. Ils correspondent à des blocs et des galets de quartzites et de grès. Ils sont enrobés dans une matrice argilosableuse.

- $\quad$ Le deuxième épisode sous forme de coulées basaltiques (fig. 4, A) surmonte la formation sédimentaire médiane. La formation médiane est une série détritique composée de conglomérats, de grès et d'argilites rouges. Elle repose en discordance de ravinement sur les conglomérats de base de la 
formation inférieure et les roches volcaniques du premier épisode. Les grès se présentent en bancs de 0,10 à $1 \mathrm{~m}$ d'épaisseur terminant les séquences conglomératiques de base ou s'interstratifient dans les argilites. Les conglomérats sont organisés en bancs métriques, constitués d'éléments inégalement façonnés qui sont des quartzites, grès, psammites, schistes, calcaires et roches volcaniques le plus souvent imbriqués et rassemblés par une matrice argile-sableuse (fig. 4, B). Une série d'accidents décrochants de direction NE-SW et à jeu dextre affecte ces roches pendant la phase compressive du deuxième épisode (Fig. 4, C).

- Le troisième épisode éruptif est constitué par des roches de même nature que les coulées basaltiques du deuxième épisode et affleurent à l'Est de Kodiat El Alama. Il se distingue cependant par son mode de gisement filonien (Fig. 4, D).

Sur les roches volcaniques du troisième épisode repose en discordance de ravinement une formation supérieure à éléments volcaniques, composée essentiellement de grès (Figure 4, E), d'argilites rouges et de conglomérats (Fig. 4, F).

Les argilites constituent l'essentiel des formations continentales de Chougrane et se mettent en place au sommet des séquences sédimentaires. Elles sont épaisses de 5 à $15 \mathrm{~m}$. 

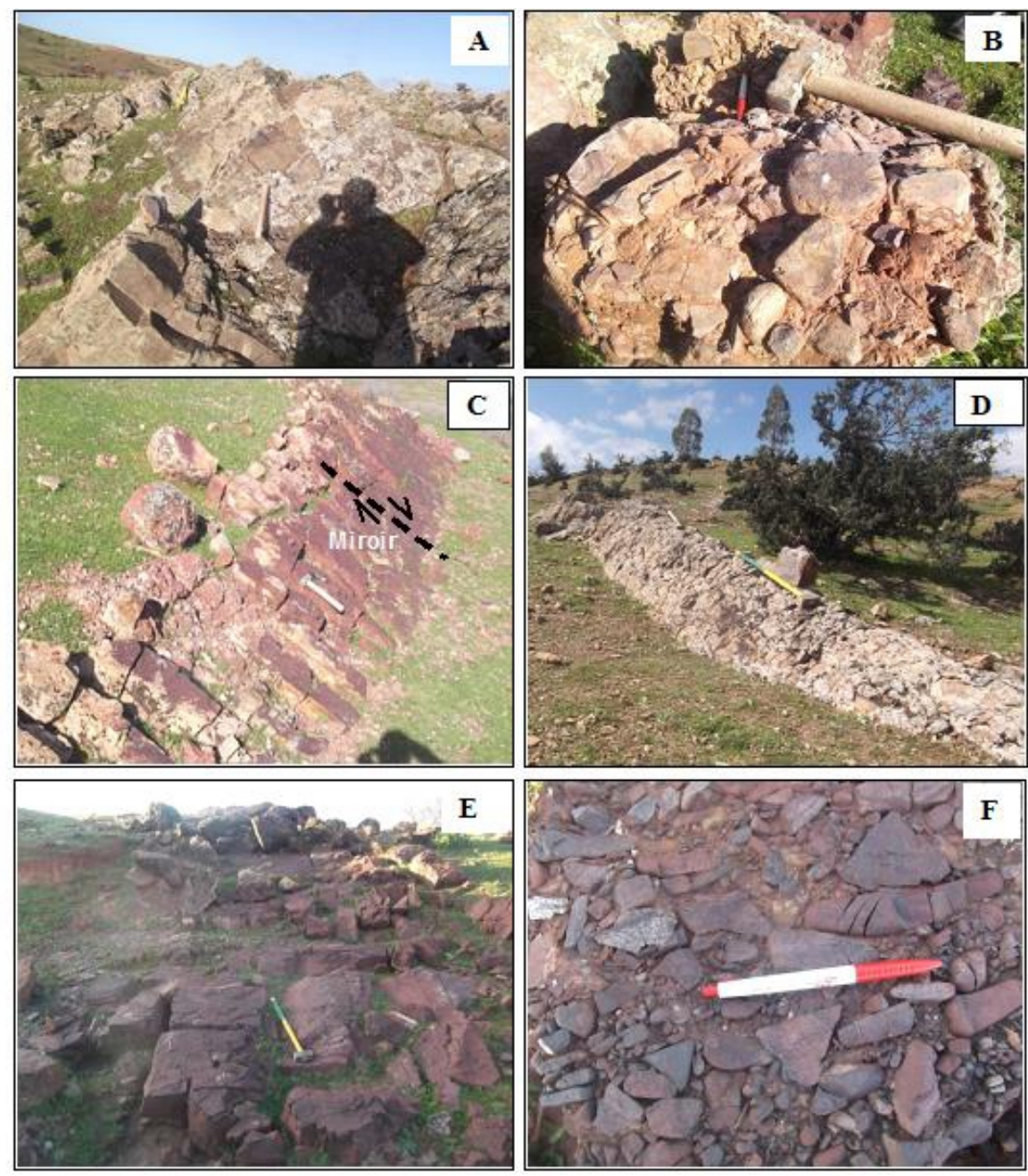

Figure 4 : Planche photographique de formations au Nord Est du bassin de Chougrane. A : coulées basaltiques du deuxième épisode, $\mathbf{B}$ : Conglomérats de formation inférieure, composés de matériel terrigène grossier mal classé, $\mathbf{C}$ : Faille décrochante de direction NE-

SW et à jeu dextre affectant les formations du deuxième épisode, $\mathbf{D}$ : basalte en filon du troisième épisode, $\mathbf{E}$ : bancs de grès de formation supérieure, $\mathbf{F}$ : Conglomérats de formation supérieure, constitués d'éléments hétérométriques inégalement façonnés rassemblés par une matrice argile sableuse. 
Etude pétrographique

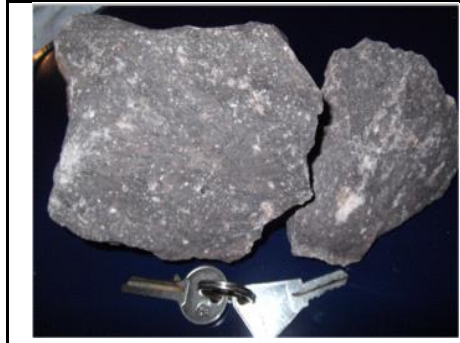

a : Rhyodacite, éch. HB2

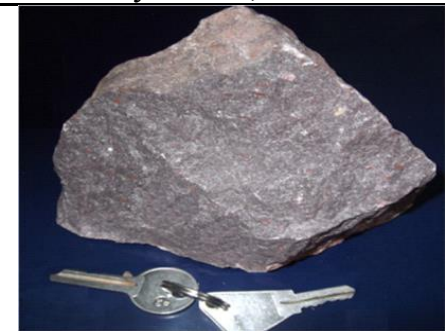

c : Basalte 1, éch. CH7

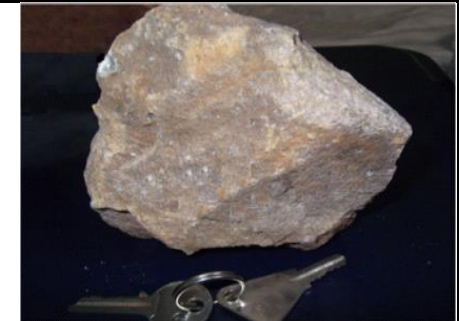

e : Basalte 2, éch. CH3
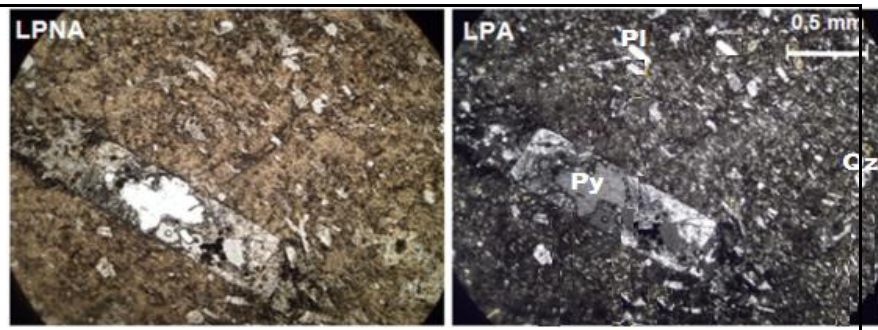

b
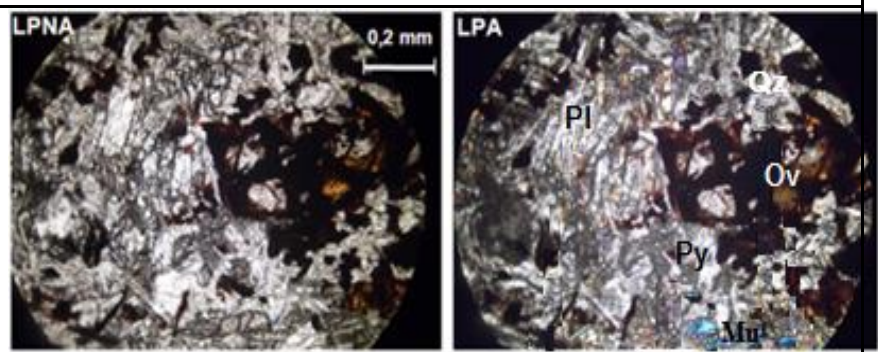

d
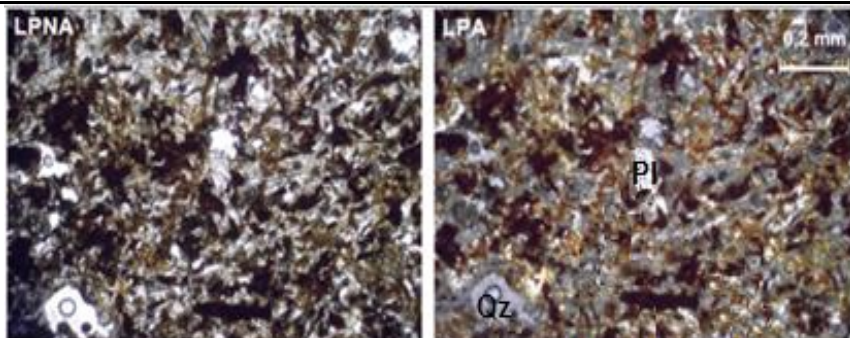

(T)

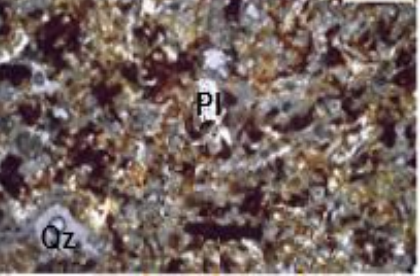

Figure 5 : Photographies des principales volcanites permiennes de Chougrane-El Had desBouhsousène.

\section{Rhyodacites}

C'est la rhyodacite échantillonnée à Tazdot, située à l'Ouest du village d'El Had des Bouhsousène. C'est une roche massive de couleur grisâtre ou brune, avec des taches blanches de plagioclase de moyennes dimensions (Fig. 5, a).

Au microscope (Fig. 5, b), la mésostase est formée essentiellement de microlites de feldspaths et de verre. Les minéraux sont généralement représentés par des phénocristaux qui sont: pyroxènes automorphes, quartz (Qz) sub-automorphes et plagioclases $(\mathrm{Pl})$ qui sont plus ou moins altérés avec formation de calcite et de séricite. On note une orientation plus ou moins nette des minéraux due à la fluidalité. 
Les rhyodacites montrent une paragenèse primaire faite de plagioclase, pyroxène et quartz et une association minérale secondaire constituée de calcite et de chlorite.

\begin{tabular}{|c|c|c|c|}
\hline & $\begin{array}{l}\text { Volcanisme permien de } \\
\text { Tiddas- Sebt Ait Ikkou }\end{array}$ & $\begin{array}{l}\text { Volcanisme permien de } \\
\text { Khénifra }\end{array}$ & $\begin{array}{c}\text { Volcanisme permien } \\
\text { de Chougrane }\end{array}$ \\
\hline faciès & $\begin{array}{c}\text { Andésite, dacites, } \\
\text { rhyolites, ignimbrites, } \\
\text { trachyte }\end{array}$ & $\begin{array}{l}\text { Andésites, rhyolites, } \\
\text { dacites, rhyodacites, } \\
\text { ignimbrites, trachyte }\end{array}$ & Rhyodacites, basaltes \\
\hline texture & $\begin{array}{c}\text { Microlitique à } \\
\text { porphyrique parfois } \\
\text { dendritique }\end{array}$ & Microlitique à porphyrique & $\begin{array}{l}\text { Microlitique à } \\
\text { porphyrique }\end{array}$ \\
\hline $\begin{array}{c}\text { paragenèse minérale } \\
\text { primaire }\end{array}$ & $\begin{array}{l}\text { quartz, feldspath, } \\
\text { biotite, Orthose }\end{array}$ & $\begin{array}{c}\text { Olivine, pyroxènes, quartz, } \\
\text { feldspath, biotite et } \\
\text { amphibole }\end{array}$ & $\begin{array}{l}\text { Olivine, pyroxènes, } \\
\text { quartz, plagioclase }\end{array}$ \\
\hline $\begin{array}{c}\text { paragenèse minérale } \\
\text { secondaire }\end{array}$ & $\begin{array}{c}\text { Chlorite, calcite, } \\
\text { séricite, Quart, opaques }\end{array}$ & $\begin{array}{c}\text { Augite, Chlorite, calcite, } \\
\text { opaques }\end{array}$ & $\begin{array}{c}\text { Chlorite, calcite, } \\
\text { quartz et muscovite }\end{array}$ \\
\hline $\begin{array}{l}\text { contexte de mise en } \\
\text { place }\end{array}$ & $\begin{array}{l}\text { la phase asturienne } \\
\text { résulte d'un régime } \\
\text { compressif a contrôlée } \\
\text { la mise en place du } \\
\text { volcanisme permien. }\end{array}$ & $\begin{array}{l}\text { régime compressif a généré } \\
\text { des éruptions volcaniques } \\
\text { qui se produisent en milieu } \\
\text { continental aérien à } \\
\text { subaérien }\end{array}$ & $\begin{array}{l}\text { régime compressif } \\
\text { guide l'ascension des } \\
\text { vulcanites (Cailleux } \\
\text { et al., 1982) }\end{array}$ \\
\hline $\begin{array}{c}\text { Références } \\
\text { bibliographiques }\end{array}$ & El Wartiti, 1990 & Youbi, 1998 & Ce travail \\
\hline
\end{tabular}

Tableau 1 : Comparaison des caractéristiques pétrographiques du volcanisme permien de Chougrane-El Had des Bouhsoussèneavec ceux des volcanismes permiens de Tiddas- Sebt Ait Ikkou et de Khénifra.

\section{Basalte (1)}

Il s'agit d'une roche très compacte, de couleur grisâtre (Fig. 5, c). En lames minces (Fig. 5, d) ces roches présentent une texture microlitique. Le basalte (1) est constitué par une paragenèse primaire faite de plagioclase $(\mathrm{Pl})$, pyroxène (Py) et olivine (Ov) et d'une association minérale secondaire faite de muscovite, calcite, de chlorite et de quartz. La matrice, très fine, est constituée de microlites de feldspaths, quartz, muscovite, calcite et chlorite en plus de verre avec oxydes.

\section{Basalte (2)}

Roche massive intensément altérée de couleur brune-grisâtre avec des phénocristaux de plagioclases (Fig. 5, e).

$\mathrm{Au}$ microscope (Fig. 5, f), ces roches présentent une texture microlitique porphyrique. Les phénocristaux sont représentés par le plagioclase. La matrice est constituée de microlites de feldspaths, quartz, calcite, chlorite, verre et oxydes.

Le basalte (2) est constituée par une paragenèse primaire dont le plagioclase est la seule relique, et d'une association minérale secondaire formée de quartz, calcite et chlorite. 


\section{Discussion et conclusions}

Même en absence de données géochimiques, on note de fortes ressemblances dans les compositions pétrographiques entre les roches volcaniques permiennes du bassin de Chougrane-El Had des Bouhsoussène et les roches volcaniques pénécontemporaines des complexes permiens de Khénifra et de Tiddas- Sebt Ait Ikkou (Maroc Central, Tableau 1). La texture des roches est en général microlitique à porphyrique. Les rhyodacites montrent une paragenèse primaire faite de plagioclase, pyroxène et quartz et une association minérale secondaire constituée de calcite et de chlorite. Les basaltes sont constitués par une paragenèse primaire faite de plagioclase, pyroxène et olivine et d'une association minérale secondaire faite de muscovite, calcite, de chlorite, quartz et opaques. Il y a absence totale de biotite et d'amphibole dans les roches que nous avons étudiées.

L'instabilité tectonique antépermienne et synpermienne sont à l'origine de grandes fractures profondes, guide la genèse du bassin et aussi l'ascension des vulcanites (Cailleux et al., 1982). Dans cette région trois épisodes volcaniques sont enregistrés, deux principaux en forme de coulées et un troisième épisode éruptif à mode de gisement filonien.

La région de Chougrane-El Had des Bouhsoussène soumise au cours du Permien à un régime essentiellement compressif a enregistrée trois épisodes volcaniques.

\section{References:}

Aït Brahim L. et Tahiri A. (1996). Rotation horaire des contraintes et mécanismes d'ouverture et de fermeture des bassins permiens du Maroc central. In Medina F. (Edit.) Le Permien et le Trias du Maroc : états des connaissances. Editions PUMAG, pp. 87-98. Marrakech.

Boushaba A., Cailleux Y. et El Wartiti M. (1987). Les granitoïdes hercyniens et le volcanisme permien du domaine mésétien marocain. Bull. Sci. Terre, Rabat, n³, pp. 104-138.

Bouya, N. (2014). Utilisation des données géophysiques aéroportées magnétiques et spectrométriques dans la cartographie géologique de la partie NE du massif central marocain et des causses moyen atlasiques. Thèse Doctorat, Univ. Moulay Ismail, Fac. Science s, 190p

Cailleux Y. (1981). Mise en évidence des de rides Ordoviciennes dans le bassin carbonifère des Bouhsousène (Maroc central). Mines et géol., Rabat (Résumés des journées géologiques, 1981).

Cailleux Y., Gonord H., LE Guern M. et Sauvage M. (1982). Sur l'âge permien du volcanisme acide tertiaire dans le Maroc central. 9éme R.A.S.T. Paris.

Cailleux Y., Gonord H., LE Guern M. et Sauvage M. (1983). Taphrogenèse et magmatisme permiens dans le Maroc Central. Bull. Fac. Sci., Marrakech. 
Vol.1, pp.24-39.

Cailleux Y., Delouche C., Gonord H., Zouine E.M. (1986). Synthèse sur le volcanisme Permien du Maroc. Son insertion dans le contexte géodynamique ouest-méditerranéen. $111^{\mathrm{e}}$ congrès national des sociétés savantes 1, 221-235. EL Wartiti, M. (1990). Le Permien du Maroc mésétien : étude géologique et implications paléogéographiques. Thèse Sciences, Univ. Mohammed $\mathrm{V}$, Rabat, 501p.

Jebrak M. (1982). Les districts à fluorine du Maroc central. Bull. B.R.G.M., 2, sect. II, n², pp. 211-221.

Le Guern M., Sauvage J.F. et Sauvage M., (1983). Dynamique du volcanisme permien dans le massif central marocain. Bull. Fac. Sci. 1, pp. 51 Marrakech.

Michard A. (1976). Eléments de géologie marocaine. Notes et mém. Serv. Géol. Maroc, $\mathrm{n}^{\circ} 252,408 \mathrm{p}$.

Piqué A. (1994). Géologie du Maroc. Les domaines régionaux et leur évolution structurale, édition Pumag, Marrakech, 284p.

Saidi A. (1996). Paléochamps de contraintes et importance de l'héritage hercynien dans la structuration de la bordure nord du Maroc central du Permien à l'actuel. Thèse de 3éme cycle. Université Mohamed V Rabat. $198 p$.

Termier H. (1936). Etude géologique sur le Maroc central et le Moyen-Atlas septentrional. Notes et mém. Serv. Mines et carte géol. Maroc, n³3, t.1, $743 p$.

Van Houten F. B. (1976). Late variscan non marine basin deposits, Northwest Africa: Record of hercynotype orogeny. In: Falke H. (ed).The continental Permian in Central, West and South Europe. NATO ASI Series, C22, pp. 215-224. Amsterdam.

Verset Y. (1988). Mémoire explicatif de la carte géologique au 100.000, Feuille de Kasbat-Tadla, notes et mém. sérv. géol. N³40 bis, Rabat, Maroc, $131 \mathrm{p}$.

Westphal M., Montigny R., Thuizat R., Bardon C., Bossert A., Hamseh A. et Rolly J.P. (1979). Paléomagnétisme et datation du volcanisme permien, triasique et crétacé du Maroc. Can. Journ. Earth. Sci., 16pp. 2150-2164.

Youbi N. (1998). Le volcanisme "post-collisionnel": un magmatisme intraplaque relié à des panaches mantelliques. Etude volcanologique et géochimique. Exemples d'application dans le Néoprotérozoïque terminal (PIII) de 1'Anti-Atlas et le Permien du Maroc. Thèse Sciences, Univ. Cadi Ayyad, Marrakech. 660p. 